\title{
Aspects pratiques en élevage d'aulacodes (Thryonomys swinderianus). VI. Mesures préventives contre la mauvaise usure des incisives
}

\author{
G.A. Mensah ${ }^{1}$ A. Schwarzenberg ${ }^{2}$ C.-H. Stier ${ }^{3}$ \\ T. Kangni ${ }^{4}$ C.F. Gall ${ }^{2 *}$
}

Mots-clés

Thryonomys swinderianus - Dent U sure - Captivité - Vitamine C - Santé animale - Bénin - Allemagne.

\section{Résumé}

Des expériences ont été conduites avec des aulacodes capturés ou nés en captivité, afin d'étudier des éléments facilitant l'usure des incisives et d'analyser l'effet de la vitamine $\mathrm{C}$ dans la ration alimentaire sur l'usure des incisives. Pour l'usure mécanique de celles-ci, l'aulacode utilise surtout le métal et le bois. L'hypothèse d'une avitaminose $C$ dans l'alimentation comme cause d'une croissance démesurée des incisives n'est pas confirmée. M ettre à la disposition des animaux des dispositifs et différents matériaux seraient une mesure indiquée pour éviter les pertes dues à des problèmes dentaires. De plus, l'ajout d'ingrédients grossiers dans la ration alimentaire et l'absence de facteurs de stress peuvent aider l'aulacode à user normalement ses incisives.

\section{INTRO DUCTION}

Comme chez tous les rongeurs, les incisives de l'aulacode ont une croissance continue. A l'état sauvage, il consomme canne à sucre (Saccharum officinarum), herbe à éléphant (Pennisetum purpureum) et autres graminées. Sa façon de manger entraîne apparemment une usure des incisives proportionnelle à sa croissance (5).

Dans la station d'élevage du Projet Bénino-Allemand d'Aulacodiculture (PBAA), seuls les aulacodes sauvages récemment capturés recevaient, avec une alimentation variée, des morceaux de canne à sucre pendant leur phase d'adaptation à la captivité. Un an

1. Unité de Recherches Zootechnique et Vétérinaire, INRA du Bénin, Ministère du Développement Rural, BP 2359, Cotonou, République du Bénin

2. Institut de Production Animale des Pays Tropicaux et Subtropicaux, Université de Hohenheim (480), Garbenstraße 17, D-70593 Stuttgart, Allemagne

3. Fachhochschule Rheinland-Pfalz, FB Agrarwirtschaft, Rochusallee 4, 55411, Bingen (am Rhein), Allemagne

4. Projet Bénino-Allemand d'Aulacodiculture, Ministère du Développement Rural, BP 03-2900, Cotonou, République du Bénin

* Auteur pour la correspondance : Tél. : 497114593170 ; Fax : 497114593290

E-mail : gall@uniho.de après la suppression de la canne à sucre, une usure insuffisante des incisives a été observée chez 67 p. 100 des animaux du cheptel (1).

L'usure insuffisante des incisives de l'aulacode engendre des pertes dans l'élevage. Ce problème serait dû à un défaut d'ajustement spontané des incisives ou à l'absence de certains éléments permettant à l'aulacode dans son biotope naturel d'entretenir ses dents, ou bien au fait que certains aulacodes n'arrivent pas à utiliser les dispositifs disponibles dans l'élevage $(1,5)$.

Comme l'homme affecté par le scorbut (causé par une carence en vitamine C) souffre de la membrane périodentaire, ce qui l'empêche de mâcher, il a paru opportun aux auteurs d'examiner l'effet de la vitamine $C$ sur l'usure des dents. L'avitaminose $C$ serait en effet responsable de la décoloration des dents et de la mauvaise croissance ou de la croissance démesurée des incisives chez le cobaye.

Bien que la plupart des mammifères et des oiseaux synthétisent la vitamine $\mathrm{C}$ dans leur foie (2), il existe d'autres animaux (jeunes oiseaux, cobayes, rats mutants et primates) et l'homme qui n'ont pas cette faculté ou bien qui l'ont perdue.

Ainsi, bien qu' $E$. coli soit présent dans la microflore intestinale de l'aulacode, il n'a pas, jusqu'à présent, été prouvé qu'il synthétise 
la vitamine C, comme c'est le cas chez d'autres animaux (8) ; par ailleurs, on ne dispose d'aucune connaissance en matière de besoins en vitamine $\mathrm{C}$ de l'aulacode.

De l'acide ascorbique est administré lors d'un traitement du scorbut. En ce qui concerne les besoins en vitamine $\mathrm{C}$, les avis sont partagés et une dose journalière de 42 à $167 \mathrm{mg} / \mathrm{kg}$ poids vif (7) ou de 150 à $200 \mathrm{mg} / \mathrm{kg}$ poids vif (6) est préconisée pour les animaux de laboratoire. En cas de stress, le besoin en vitamine $\mathrm{C}$ serait plus élevé (4), et certains animaux en synthétiseraient plus (7).

Le présent article expose des études sur des dispositifs et des éléments facilitant l'usure des incisives, ainsi que des mesures préventives prises pour pallier aux effets d'une usure insuffisante.

\section{MATERIEL ET METHODES}

Les études ont été faites dans les élevages d'aulacodes du Projet Bénino-Allemand d'Aulacodiculture à Godomey (Bénin) et de l'Université de Hohenheim (Allemagne). Les aulacodes étaient élevés individuellement en cage hors sol ou en enclos au sol en groupe de reproduction. A l'Université de Hohenheim, les animaux étaient gardés dans un bâtiment chauffé, ventilé et obscur où des conditions d'éclairage de jour étaient simulées de 23 à $11 \mathrm{~h}$ tout au long de l'année.

Quatre expériences différentes ont été conduites :

\section{Première expérience : inventaire de quelques dispositifs et matériaux facilitant l'usure des incisives}

L'expérience a été conduite au PBAA et a duré quatre mois avec trois groupes d'animaux :

- 509 aulacodes (dont 230 mâles) récemment capturés à l'état sauvage ;

- 571 aulacodes (dont 300 mâles) en captivité depuis un an ;

- 116 aulacodes (dont 52 mâles) nés en captivité et faisant partie de l'expérience dès l'âge de 4 jours.

Les animaux étaient nourris avec des grains de maïs sec, des granulés de concentré, de la canne à sucre (Saccharum officinarum) et du paspalum frais (Paspalum vaginatum).

Les dispositifs ou matériaux suivants ont été installés dans les cages et enclos d'élevage pour les 1196 animaux :

- grillages à mailles carrées de 2,5 cm de côté, diamètre du fil $1,8 \mathrm{~mm}$;

- fils de fer (2,45 mm de diamètre) placés en trois lignes parallèles et horizontales, l'un au-dessus de l'autre, espacés entre eux de $10 \mathrm{~cm}$;

- fils de fer $(2,45 \mathrm{~mm}$ de diamètre) de suspension des bottes de fourrages ;

- abreuvoirs-biberons avec tétines métalliques ;

- morceaux de roches cristallines : granit, gneiss et galet ;

- bouts de bois d'acajou (Khaya senegalensis) ;

- coques de noix de coco (Cocos nucifera).

Les dispositifs les plus utilisés étaient observés trois fois par jour entre 10 et 12 h, 16 et 18 h, ainsi que 22 et 24 h. De même, au cours de l'inspection du cheptel et de la conduite de l'élevage de 7 à $10 \mathrm{~h}$, tous les aulacodes observés s'ajustant les dents sur l'un des dispositifs étaient enregistrés.
Pour quantifier l'utilisation d'un dispositif donné, les critères suivants ont été fixés :

- dispositif peu utilisé : temps d'utilisation par l'animal inférieur à une demi-minute ;

- dispositif moyennement utilisé : temps d'utilisation par l'animal d'environ une minute ;

- dispositif très utilisé : temps d'utilisation par l'animal supérieur à une minute et demi.

Les incisives des animaux récemment capturés ont été inspectées à leur arrivée et à leur sortie de la quarantaine au bout de trois semaines. Celles des aulacodeaux (jeunes aulacodes) furent inspectées le $4^{\mathrm{e}}$ jour après leur naissance et à leur sevrage au $28^{\mathrm{e}}$ jour. L'inspection des incisives de tous les animaux de l'expérience était faite au cours des pesées mensuelles, des traitements sanitaires et des transferts.

\section{Deuxième expérience : influence du diamètre du granulé de concentré sur l'usure des incisives de l'aulacode}

L'expérience a été conduite à l'Université de Hohenheim et a duré 20 semaines dont 4 semaines de phase de transition alimentaire et d'adaptation. Vingt-neuf aulacodes mâles, adultes, nés en captivité et d'âge moyen de 9 mois, ont été utilisés. Ils étaient élevés en cage individuelle et abreuvés à volonté. Les animaux étaient répartis en trois groupes, l'un de 11 animaux et les deux autres de 9. Ils étaient nourris avec des granulés de trois diamètres différents : 4, 6 et $8 \mathrm{~mm}$.

Les ingrédients utilisés pour fabriquer le granulé étaient :

$\begin{array}{lr} & \text { p. } 100 \\ \text { Tourteaux de soja } & 30,0 \\ \text { Blé } & 27,5 \\ \text { Seigle } & 12,5 \\ \text { Avoine } & 15,0 \\ \text { Paille } & 7,5 \\ \text { Huile de soja } & 3,0 \\ \text { CMV } & 4,5\end{array}$

La composition du granulé (92,3 p. 100 de matière sèche (MS)), selon l'analyse de Weende, était la suivante :

$$
\text { p. } 100 \text { de MS }
$$

Protéines brutes

Lipides brutes

Fibres brutes

Extractif non azoté

Cendres

L'état des incisives a été contrôlé au début et à la fin de l'expérience ; trois indices ont été retenus (1):

- indice 0 : incisives sans défaut ;

- indice 1 : quelques défauts mais l'état des incisives ne gênait pas l'animal pour s'alimenter normalement ;

- indice 2 : l'état des incisives ne permettait plus à l'animal de s'alimenter correctement. 
Le gaspillage alimentaire a été calculé comme fraction gaspillée sur la quantité de granulés donnée. Les animaux ont été pesés au début et à la fin de l'expérience.

\section{Troisième expérience : influence de la vitamine $C$ et de trois sortes de bois différents sur l'usure des incisives de l'aulacode sauvage capturé}

L'expérience a été conduite à l'Université de Hohenheim et a duré cinq mois. Dix-neuf aulacodes mâles, sauvages, capturés et répartis en deux groupes de 10 et 9 animaux, ont été utilisés. Ils étaient élevés en cage individuelle, nourris et abreuvés à volonté :

- groupe 1 (témoin) : foin et granulé sans vitamine C ;

- groupe 2 : foin et granulé contenant 0,1375 p. 100 de vitamine C.

Trois sortes de bouts de bois leur étaient régulièrement distribués : bois de saule (bois tendre), bois de hêtre (bois dur) et bois de hêtre imprégné de glucose (bois glucosé). Le bois glucosé était obtenu en plongeant le bois frais dans une solution saturée de glucose à $120^{\circ} \mathrm{C}$ pendant une heure à l'étuve, puis en le faisant sécher à $40^{\circ} \mathrm{C}$ pendant une heure dans un four.

Les morceaux de bois mis dans les cages de chaque animal étaient pesés trois fois par semaine et complétés ou renouvelés au besoin. Le même bois était différencié selon le degré de séchage en bois frais et en bois sec. L'état des incisives a été contrôlé suivant trois indices comme dans la $2^{\mathrm{e}}$ expérience.

De plus, les paramètres suivants ont été étudiés :

- couleur des incisives : jaunes ou blanches ;

- utilisation des morceaux de bois (perte de poids du bois, en g) ;

- développement du poids vif des animaux (en g).

\section{Quatrième expérience : influence de la vitamine $C$ et de trois sortes de bois différents sur l'usure des incisives de l'aulacode né en captivité}

L'expérience a été conduite à l'Université de Hohenheim ; elle a duré 12 semaines.

Vingt-sept aulacodes mâles nés en captivité et élevés dans des cages individuelles ont été utilisés. Ils ont été répartis en quatre groupes expérimentaux de 6 ou 7 animaux, nourris avec des granulés durs ou tendres, avec ou sans vitamine $\mathrm{C}$, et du bois à ronger (pour user leurs incisives) leur a été donné selon les dispositifs indiqués au tableau I.

Le granulé a été durci en ajoutant 5 p. 100 de mélasse de canne à sucre. En revanche, le granulé dit tendre a été fabriqué sans mélasse.

- groupe 1 (témoin) : recevait des granulés durs avec de la vitamine $\mathrm{C}(0,1375$ p. 100$)$ et trois sortes de bois ;

- groupe 2 : recevait trois sortes de bois, des granulés durs mais pas de vitamine $\mathrm{C}$;

- groupe 3 : recevait des granulés durs avec de la vitamine C $(0,1375$ p. 100) mais pas de bois ;

- groupe 4 : recevait trois sortes de bois, de la vitamine C $(0,1375$ p. 100), des granulés tendres mais pas de granulés durs.

Trois sortes de bois ont été utilisés pour éviter un effet éventuel de leur qualité ou autre. Ils étaient identiques à ceux de la $3^{\mathrm{e}}$ expérience,

\section{Tableau I}

Utilisation des dispositifs facilitant I'usure des incisives de l'aulacode ( $\%$ d'observations, $\mathrm{n}=920$ )

\begin{tabular}{|c|c|c|c|}
\hline & $\begin{array}{l}\text { Dec } \\
\text { peu }^{1}\end{array}$ & $\begin{array}{l}\text { ré d'utilise } \\
\text { moyen }^{2}\end{array}$ & $\begin{array}{l}\text { tion } \\
\text { élevé }^{3}\end{array}$ \\
\hline Grillage à mailles carrées & 1,09 & 18,48 & 21,74 \\
\hline $\begin{array}{l}\text { Fils métalliques en rangées } \\
\text { horizontales et parallèles }\end{array}$ & 2,17 & 16,30 & 19,56 \\
\hline $\begin{array}{l}\text { Embout de la tétine } \\
\text { de l'abreuvoir-biberon }\end{array}$ & 1,09 & 6,52 & 4,35 \\
\hline $\begin{array}{l}\text { Fil de fer de suspension } \\
\text { de la botte de fourrages }\end{array}$ & - & 2,17 & 4,35 \\
\hline Bout de bois d'acajou & 1,09 & 1,09 & - \\
\hline Coque de noix de coco & - & - & - \\
\hline $\begin{array}{l}\text { Morceaux de roches } \\
\text { cristallines }\end{array}$ & - & - & - \\
\hline
\end{tabular}

bois de saule (bois tendre), bois de hêtre (bois dur) et bois de hêtre imprégné dans une solution saturée de glucose (bois glucosé). Ils ont été mis à la disposition des aulacodes comme dans l'expérience précédente. Les granulés tendres étaient préparés en ajoutant de l'eau pendant la granulation.

L'état des incisives a été contrôlé selon trois indices comme dans la $2^{\mathrm{e}}$ expérience. Outre les trois paramètres retenus lors de la $3^{\mathrm{e}}$ expérience, les paramètres suivants ont été étudiés :

- mesure des incisives : longueur, largeur (5) et, comme profondeur, le creux de l'encoche des incisives supérieures dans lequel viennent se loger les incisives du bas ;

- surcroît des incisives (différence entre croissance et usure).

Les mensurations des incisives ont été faites à l'aide d'un pied à coulisse (5). Pour effectuer les mesures, les animaux ont été placés sous anesthésie générale.

Les mesures linéaires des incisives et les prises de poids vif ont été effectuées au début de l'expérience, puis après 6 et 12 semaines.

\section{RESU LTATS}

\section{Première expérience}

Tous les animaux sauvages capturés et les aulacodeaux avaient, sans exception, des incisives jaune orangé et sans défaut.

Parmi les aliments distribués, c'était la canne à sucre qui était la première consommée, puis c'était le tour du granulé et enfin celui du maïs. La coque de noix de coco et les morceaux de roches cristallines n'ont pas été utilisés par les animaux durant toute la période d'observation. Les aulacodes avaient d'abord recours au métal et, à défaut de métal, au bois (tableau I). 


\section{Deuxième expérience}

La plupart des aulacodes des trois groupes avaient des dents sans défaut : dans chaque groupe il n'a été trouvé qu'un faible pourcentage d'animaux avec des incisives mal usées et les différences n'étaient pas significatives ( $p \geq 0,05)$ (tableau II).

Tous les animaux dans les trois groupes ont gagné du poids et les différences entre les trois groupes n'étaient pas significatives. Le gaspillage alimentaire a été respectivement : 6,8 p. 100 (granulé $4 \mathrm{~mm}$ ), 39,7 p. 100 (granulé $6 \mathrm{~mm}$ ) et 49 p. 100 (granulé $8 \mathrm{~mm}$ ). Le gaspillage du granulé de diamètre $4 \mathrm{~mm}$ est significativement plus faible $(\mathrm{p} \leq 0,01)$ que celui des deux autres.

\section{Tableau II}

Etat des incisives des aulacodes en fonction du diamètre du granulé (\% d'observations)

\begin{tabular}{cccc} 
& \multicolumn{3}{c}{ Diamètre du granulé* } \\
\cline { 2 - 4 } Etat des incisives & $\begin{array}{c}\mathbf{4} \mathbf{~ m m} \\
(\mathbf{n}=\mathbf{9})\end{array}$ & $\begin{array}{c}\mathbf{6} \mathbf{~ m m} \\
(\mathbf{n}=\mathbf{9})\end{array}$ & $\begin{array}{c}\mathbf{8} \mathbf{~ m m} \\
(\mathbf{n}=\mathbf{1 1})\end{array}$ \\
0 & 66,67 & 88,89 & 81,82 \\
1 & 11,11 & 00,00 & 00,00 \\
2 & 22,22 & 11,11 & 18,18
\end{tabular}

$0=$ sans défaut

$1=$ quelques défauts mais alimentation possible

$2=$ mauvais état et alimentation difficile

* les différences attribuées au diamètre du granulé ne sont pas significativement différentes $(p \geq 0,05)$

\section{Troisième expérience}

Les animaux du groupe 1 (sans vitamine $\mathrm{C}$ ) avaient des incisives plus longues que ceux du groupe 2, mais les différences n'étaient pas significatives $(\mathrm{p} \geq 0,05)$ (tableau III). Aucun problème dentaire n'a été observé chez les aulacodes pendant les cinq mois d'observation.

\section{Tableau III}

Longueur des incisives et gain pondéral des aulacodes

\begin{tabular}{lcc} 
& $\begin{array}{c}\text { Groupe 1 } \\
\text { (vitamine } \mathbf{C}) * \\
(\mathbf{n}=\mathbf{9})\end{array}$ & $\begin{array}{c}\text { Groupe 2 } \\
\text { (témoin)* } \\
(\mathbf{n}=\mathbf{1 0})\end{array}$ \\
\hline $\begin{array}{l}\text { Longueur des incisives } \\
\text { - supérieures }(\mathrm{mm})\end{array}$ & $9,2 \pm 1,7$ & $11,2 \pm 1,9$ \\
- inférieures $(\mathrm{mm})$ & $8,7 \pm 1,5$ & $11,6 \pm 2,4$ \\
Poids & & \\
- initial (g) & $3098,0 \pm 50,7$ & $3096,0 \pm 31,5$ \\
- final (g) & $3916,8 \pm 197,0$ & $3973,4 \pm 99,7$ \\
Age (jours) & $352,0 \pm 3,3$ & $352,0 \pm 3,9$ \\
Gain de poids total (g) & $818,8 \pm 166,3$ & $877,4 \pm 95,3$
\end{tabular}

* Les valeurs moyennes entre les deux groupes ne sont pas significativement différentes $(\mathrm{p} \geq 0,05)$
Les gains de poids du groupe avec vitamine $\mathrm{C}$ étaient plus élevés que ceux du groupe contrôle, mais la différence n'était pas significative $(\mathrm{p} \geq 0,05)$.

La quantité de bois glucosé rongé était significativement plus élevée $(\mathrm{p} \leq 0,05)$ dans les deux groupes que celle des bois tendre et dur (tableau IV).

\section{Tableau IV}

Utilisation des trois sortes de bois (en g) chez les aulacodes de la $3^{e}$ expérience (valeurs moyennes du moindre carré*)

\begin{tabular}{lcc} 
& $\begin{array}{c}\text { Groupe 1 } \\
\text { (témoin) } \\
(\mathbf{n}=\mathbf{9})\end{array}$ & $\begin{array}{c}\text { G roupe 2 } \\
\text { (vitamine } \mathbf{C}) \\
(\mathbf{n}=\mathbf{1 0})\end{array}$ \\
\hline $\begin{array}{l}\text { Bois tendre } \\
\text { Bois dur }\end{array}$ & $139,1^{\mathrm{a}} \pm 220,4$ & $151,5^{\mathrm{a}} \pm 258,7$ \\
Bois glucosé & $102,1^{\mathrm{a}} \pm 110,2$ & $176,4^{\mathrm{a}} \pm 269,3$ \\
& $307,4^{\mathrm{b}} \pm 418,3$ & $302,2^{\mathrm{b}} \pm 518,0$
\end{tabular}

* Les valeurs moyennes suivies de lettres différentes sur une même colonne sont significativement différentes $(\mathrm{p} \leq 0,05)$

\section{Quatrième expérience}

Dans le tableau V sont indiquées les dimensions moyennes (longueur, largeur et profondeur) des incisives supérieures et inférieures. Les différences observées à leur niveau entre les animaux des quatre groupes ne sont pas significatives $(p \geq 0,05)$. Le tableau VI résume le surcroît enregistré chez les animaux dans chaque groupe. Les différences entre les groupes sont plus importantes pour le surcroît des incisives inférieures que pour celui des incisives supérieures, mais elles ne sont pas significatives $(p \geq 0,05)$.

Dans le tableau VII est montrée la quantité des trois sortes de bois rongé par les aulacodes, dans les trois groupes qui en ont reçu. Il existe une relation entre le degré de séchage du bois, le traitement $d u$ groupe $(p \leq 0,01)$, puis la sorte de bois $(p \leq 0,001)$. Aucune relation n'existe entre la sorte de bois et le surcroît des incisives $(p \geq 0,05)$. Les animaux qui préféraient des morceaux de bois dur avaient une usure plus forte de leurs incisives, alors que les animaux qui rongeaient le plus de bois (groupe 2) avaient le surcroît le plus élevé (tableau VI).

Il n'y a pas de différences significatives $(\mathrm{p} \geq 0,05)$ au niveau des paramètres étudiés entre les groupes 1,2 et 3 qui ont reçu le granulé dur et le groupe 4 le granulé tendre. La dureté n'a donc eu aucune incidence sur l'usure des incisives.

Une classification des animaux selon la couleur des dents ou le comportement n'a pas relevé de différences de surcroît des incisives ni de gain pondéral.

\section{DISCUSSION}

Les dents des animaux capturés ainsi que celles des jeunes nés en captivité étaient en parfaite condition. Dans la nature, l'aulacode consomme des fourrages qui sont riches en cellulose et parfois en lignine et silice (feuilles et tiges des graminées). Ces aliments constituent à la fois un matériau énergétique, un lest et une véri- 


\section{Tableau V}

Dimensions moyennes $\left(x \pm s_{x}\right)$ des incisives (en $\mathrm{mm}$ )

\begin{tabular}{|c|c|c|c|c|c|}
\hline & $\begin{array}{c}\text { G roupe } 1 \\
\text { (témoin) } \\
(\mathrm{n}=7)\end{array}$ & $\begin{array}{c}\text { G roupe } 2 \\
\text { (sans vitamine C) } \\
(\mathbf{n}=7)\end{array}$ & $\begin{array}{c}\text { Groupe } 3 \\
\text { (sans bois) } \\
(\mathrm{n}=6)\end{array}$ & $\begin{array}{c}\text { Groupe } 4 \\
\text { (granulés tendres) } \\
(\mathrm{n}=7)\end{array}$ & $\begin{array}{l}\text { Total } \\
(n=27)\end{array}$ \\
\hline \multicolumn{6}{|l|}{ Supérieures } \\
\hline - longueur & $10,8 \pm 2,2$ & $11,1 \pm 1,6$ & $12,8 \pm 1,2$ & $11,3 \pm 1,6$ & $11,4 \pm 1,8$ \\
\hline - largeur & $10,9 \pm 2,5$ & $11,7 \pm 0,6$ & $11,9 \pm 0,4$ & $11,9 \pm 0,5$ & $11,6 \pm 1,4$ \\
\hline - profondeur & $4,9 \pm 0,4$ & $5,1 \pm 0,4$ & $5,2 \pm 0,3$ & $5,3 \pm 0,3$ & $5,1 \pm 0,4$ \\
\hline \multicolumn{6}{|l|}{ Inférieures } \\
\hline - Iongueur & $13,2 \pm 1,4$ & $13,8 \pm 1,9$ & $12,2 \pm 2,3$ & $12,0 \pm 1,8$ & $12,8 \pm 2,0$ \\
\hline - largeur & $11,2 \pm 0,6$ & $11,6 \pm 0,4$ & $11,8 \pm 0,6$ & $11,9 \pm 0,4$ & $11,6 \pm 0,6$ \\
\hline - profondeur & $4,7 \pm 0,4$ & $5,0 \pm 0,4$ & $5,1 \pm 0,3$ & $4,9 \pm 0,4$ & $4,9 \pm 0,4$ \\
\hline
\end{tabular}

Tableau VI

Surcroît des incisives supérieures et inférieures dans chaque groupe (en $\mathrm{mm}$ ) (valeurs moyennes du moindre carré)

\begin{tabular}{lcccc} 
& $\begin{array}{c}\text { G roupe 1 } \\
\text { (ttémoin) } \\
(\mathbf{n}=\mathbf{7})\end{array}$ & $\begin{array}{c}\text { Groupe 2 } \\
\text { (sans vitamine C) } \\
(\mathbf{n}=\mathbf{7})\end{array}$ & $\begin{array}{c}\text { Groupe 3 } \\
(\text { sans bois) } \\
(\mathbf{n}=\mathbf{6})\end{array}$ & $\begin{array}{c}\text { G roupe 4 } \\
\text { (granulés tendres) } \\
(\mathbf{n}=\mathbf{7})\end{array}$ \\
\hline Surcroît supérieur & $1,32 \pm 0,47$ & $1,75 \pm 0,52$ & $1,45 \pm 0,53$ & $1,57 \pm 0,42$ \\
Surcroît inférieur & $0,80 \pm 0,43$ & $1,22 \pm 0,47$ & $0,22 \pm 0,45$ & $-0,16 \pm 0,44$
\end{tabular}

Tableau VII

Quantité de bois rongé par semaine (en g) (valeurs moyennes du moindre carré*) **

\begin{tabular}{lccr} 
& $\begin{array}{c}\text { G roupe 1 } \\
\text { (témoin) } \\
\mathbf{n = 7}\end{array}$ & $\begin{array}{c}\text { Groupe 2 } \\
\text { (sans vitamine C) } \\
\mathbf{n}=\mathbf{7}\end{array}$ & $\begin{array}{c}\text { Groupe 4 } \\
\text { (granulés tendres) } \\
\mathbf{n}=\mathbf{7}\end{array}$ \\
\hline $\begin{array}{l}\text { Bois tendre } \\
\text { Bois dur }\end{array}$ & $123,8^{\mathrm{a}} \pm 69,5$ & $224,2^{\mathrm{b}} \pm 69,5$ & $40,2^{\mathrm{a}} \pm 69,5$ \\
Bois glucosé & $103,2^{\mathrm{a}} \pm 69,5$ & $355,1^{\mathrm{b}} \pm 69,5$ & $105,4^{\mathrm{a}} \pm 69,5$ \\
Bois frais & $149,6^{\mathrm{a}} \pm 69,5$ & $663,4^{\mathrm{c}} \pm 69,5$ & $152,6^{\mathrm{a}} \pm 69,5$ \\
Bois sec & $309,0^{\mathrm{c}} \pm 86,7$ & $677,1^{\mathrm{d}} \pm 86,7$ & $261,0 \pm 86,7$ \\
& $46,7 \pm 86,7$ & $219,9 \pm 86,7$ & $41,1 \pm 86,7$
\end{tabular}

* les valeurs moyennes suivies de lettres différentes sur une même ligne sont significativement différentes $(\mathrm{p} \leq 0,05)$

** des différences entre les écarts-types n'interviennent qu'à partir du 3 e chiffre après la virgule

table lime à user les dents. De même, l'aulacode ingère de la terre avec les racines et les tubercules qu'il déterre, en mangeant directement du sable, en grignotant des termitières et des roches tendres. L'aulacode paraît avoir conscience de la croissance rapide de ses incisives, et use celles du haut contre celles du bas en faisant des mouvements de haut en bas.

En captivité, il les frotte sur le fil de fer des mailles des grillages de sa cage d'élevage. En effet, les dispositifs les plus utilisés (après les aliments comme la canne à sucre) sont ceux de nature métallique (tableau I). De même, il a été observé que des aulacodes élevés en groupe en enclos rongeaient régulièrement les lamelles de bois apposées contre les parois latérales de séparation des enclos, dans un effort pour rejoindre ceux qui se trouvaient dans les enclos voisins. Par ailleurs, ils rongeaient régulièrement du bois, de préférence glucosé. L'entretien de leurs incisives a pu ainsi se faire et aucun problème dentaire n'a été décelé.

L'usure des incisives n'était pas augmentée par le diamètre du granulé. Comme le gaspillage alimentaire est plus important avec des granulés de gros diamètre, les granulés de diamètre plus petit sont donc à préférer.

Un effet du dosage de vitamine $\mathrm{C}$ sur les problèmes dentaires n'a pas été observé. Les aulacodes du groupe sans vitamine $\mathrm{C}$ avaient des incisives plus longues et un gain pondéral plus élevé, mais ils rongeaient plus intensivement le bois. Par conséquent, on peut 
supposer que les aulacodes n'ont pas besoin de supplémentation en vitamine $\mathrm{C}$ comme c'est le cas chez d'autres rongeurs (8). Il serait cependant souhaitable de mener des expériences plus approfondies sur le sujet.

L'émail de la face labiale des incisives des aulacodes a une coloration régulière jaune à orangée, caractéristique des rongeurs. Dans cette étude, une partie des animaux dont les incisives manquaient de coloration avaient un surcroît inférieur, bien que la différence ne soit pas statistiquement significative. Ces observations concordent avec celles de Granados (3) qui a observé que les dents pigmentées étaient plus longues que les blanches.

Le surcrôt journalier enregistré dans la $4^{\mathrm{e}}$ expérience est supérieur à celui enregistré dans la $3^{\mathrm{e}}$. Cependant, aucun problème dû aux incisives trop longues n'a été rencontré durant toute l'expérimentation. De plus, tous les aulacodes de cette $4 \mathrm{e}$ expérience avaient des gains de poids réguliers et appréciables.

La comparaison du groupe 4 avec le groupe témoin montre que la consistance des granulés n'a pas d'influence sur le surcroît des incisives. Ce résultat concorde avec celui observé dans la $2^{\mathrm{e}}$ expérience relative à la taille du granulé, qui n'avait aucune influence sur l'usure des incisives. Le groupe 3, qui n'a pas reçu de bois, présente un surcroît légèrement supérieur à celui du groupe témoin, bien que la différence observée ne soit pas statistiquement différente. Ces animaux affilent leurs incisives sur les mailles métalliques plus fréquemment que les autres qui ont du bois à leur disposition.

Les aulacodes préfèrent le bois frais au bois sec. Cependant, le bois imprégné au préalable de glucose était rongé par les animaux, même à l'état sec. Il correspondrait donc à la canne à sucre qu'il peuvent trouver au Bénin. La supplémentation en canne à sucre devrait être conservée pour faciliter l'auto-usure des incisives chez les aulacodes.

La fréquence de la mauvaise usure et des dents longues, nettement plus faible dans l'élevage expérimental en Allemagne que dans celui du Bénin, pourrait s'expliquer par une conduite de l'élevage caractérisée en Allemagne par l'absence de facteurs de stress perturbant l'actogramme des animaux.

\section{Summary}

Mensah G .A., Schwarzenberg A., Stier C.-H., Kangni T., Gall C.F. Practical aspects of grass-cutter (Thryonomys swinderianus) breeding. VI. Preventive measures against insufficient incisor wear

Experiments were carried out with grass-cutters captured or born in captivity in order to study the elements facilitating incisor attrition and to analyze the effect of vitamin $\mathrm{C}$ addition to daily rations on incisor wear. The grass-cutters mainly used metal and wood for mechanical incisor wear. The hypothesis that an excessive incisor growth was caused by avitaminosis C was not confirmed. Losses due to dental problems could be prevented by providing animals with devices and various materials. In addition, coarse materials included into the diet and a non stressful environment might help ensure proper incisor wear.

Key words: Thryonomys swinderianus - Tooth - Wear Captivity - Vitamin C - Animal health - Benin - Germany.

\section{- CONCLUSION}

Des possibilités assez simples peuvent réduire les problèmes causés par l'usure insuffisante des incisives des aulacodes, en mettant à la disposition des animaux des supports et des dispositifs leur permettant de se «faire les dents». De plus, il semble qu'une alimentation diversifiée et un élevage laissant assez de temps de repos à l'aulacode, aident à réduire les problèmes dentaires.

\section{BIBLIO GRAPHIE}

1. ADJANOHOUN E., 1986. Bilan de l'état des incisives de tous les aulacodes entretenus au PBAA au 6 septembre 1986. Cotonou, Bénin, Ministère du Développement Rural, PBAA/DEP/MDRAC/BEN IN. (Rapport technique inédit)

2. CHATTERJEE I.B., MAJU M BER A.D., NANDI B.K., SUBRAMANIAN N., 1975. Synthesis and some major functions of vitamin $C$ in animals. Ann. N.Y. Acad. Sci., $258: 24-26$.

3. GRANADOSH., 1986. Physiological role of the pigment of the enamel in the rodent incisors. Its importance in experimental biology and medicine. Arch. Invest. Med. Mex., 17 : 37-54.

4. KALLNER A., 1987. Requirement for vitamin C based on metabolic studies. In: Burns J.J., Rivers J.M., Machlin L.J. eds., Third conference on vitamin C, New York, USA, october 8-10, 1986. Ann. N.Y. Acad. Sci., 498: 418-423.

5. MENSAH G.A., BRÖNNEMANN A., STIER C.-H., GALL C.F., 1992. Aspects pratiques en élevage d'aulacodes (Thryonomys swinderianus). V. Croissance et usure normales des incisives. Revue Elev. Méd. vét. Pays trop., 45 : 175-178.

6. PAU LIN G L., 1982. Effect of dietary ascorbic acid on the incidence of spontaneous mammary tumors in R III mice. In: Hanck A., ed., Vitamin C. Bern, Switzerland, Huber, p. 7.

7. STONE I., 1977. The healing factor vitamin $C$ against disease. $\mathrm{New}$ York, USA, Grosset and Dunlap.

8. WALKER E.P., 1968. Mammals of the world, 2nd ed., Vol. II. Baltimore, USA, The Johns Hopkins Press.

Reçu le 16.2.93, accepté le 18.4.97

\section{Resumen}

Mensah G.A., Schwarzenberg A., Stier C.-H., Kangni T., G all C.F. Aspectos prácticos de la crianza del cobayo aulacodes (Thryonomys swinderianus). VI. Medidas preventivas contra el mal desgaste de los incisivos

Los estudios se realizaron con aulacodes capturados 0 nacidos en cautiverio, con el fin de estudiar los elementos que favorecen el desgaste de los incisivos, así como analizar el efecto de la vitamina $C$ en la ración alimenticia sobre el desgaste de los incisivos. Para el desgaste mecánico de éstos, el aulacodes utiliza sobre todo el metal y la madera. No se confirma la hipótesis de una avitaminosis C en la alimentación, como causa de un crecimiento desmesurado de los incisivos. Poner a disposición de los animales diferentes dispositivos y materiales, sería una medida indicada para evitar la perdidas debidas a los problemas dentarios. Además, la adición de ingredientes bruscos en la ración alimenticia y la ausencia de factores de stress pueden ayudar al aulacodes a utilizar normalmente sus incisivos.

Palabras clave: Thryonomys swinderianus - Diente - Desgaste Cautividad - Vitamina C - Sanidad animal - Benin - Alemania. 